\title{
Overexpression of circAtp9b in ulcerative colitis is induced by lipopolysaccharides and upregulates PTEN to promote the apoptosis of colonic epithelial cells
}

\author{
FENGDONG LI ${ }^{1}$, JINJIN FU ${ }^{1}$, LI FAN ${ }^{1}$, SHUANGSHUANG LU ${ }^{2}$, HUAHUI ZHANG ${ }^{2}$, \\ XIAOYING WANG ${ }^{3}$ and ZHANJU LIU ${ }^{4}$
}

\begin{abstract}
${ }^{1}$ Department of Gastroenterology, The Affiliated Changzhou No.2 People's Hospital of Nanjing Medical University,
Changzhou, Jiangsu 213003; ${ }^{2}$ Department of Internal Medicine, Graduate School of Dalian Medical University,

Dalian, Liaoning 116044; ${ }^{3}$ Department of Oncology, The Affiliated Changzhou No.2 People's Hospital of Nanjing Medical University, Changzhou, Jiangsu 213003; ${ }^{4}$ Department of Gastroenterology, Shanghai Tenth People's Hospital, Clinical Medical College of Nanjing Medical University, Shanghai 200072, P.R. China
\end{abstract}

Received September 18, 2020; Accepted August 18, 2021

DOI: $10.3892 /$ etm.2021.10840

\begin{abstract}
It has been reported that knockdown of circular RNA (circ) ATPase class II type 9B (Atp9b) can reduce lipopolysaccharide (LPS)-induced inflammation, which plays a notable role in ulcerative colitis (UC). The present study aimed to explore the role of circAtp9b in UC. The expression levels of Atp9b and PTEN in the plasma of patients with UC $(n=60)$ and healthy controls $(n=60)$ were determined via reverse transcription-quantitative PCR. Overexpression of circAtp9b and PTEN were achieved in human colonic epithelial cells (HCnEpCs) to explore the relationship between circAtp9b and PTEN. The role of circAtp9b and PTEN in regulating the apoptosis of HCnEpCs under LPS treatment was evaluated using flow cytometry. The present study revealed that circAtp9b was upregulated in UC and that it was positively correlated with PTEN. In HCnEpCs, LPS treatment resulted in upregulation of circAtp9b in a dose-dependent manner. Moreover, overexpression of circAtp9b mediated the upregulation of PTEN in HCnEpCs, while silencing of circAtp9b decreased the expression levels of PTEN. Apoptosis analysis demonstrated that overexpression of circAtp9b and PTEN promoted the apoptosis of HCnEpCs. In addition, silencing of
\end{abstract}

Correspondence to: Dr Xiaoying Wang, Department of Oncology, The Affiliated Changzhou No.2 People's Hospital of Nanjing Medical University, 29 Xinglong Lane, Tianning, Changzhou, Jiangsu 213003, P.R. China

E-mail: xiaoyingwangjiangs@163.com

Dr Zhanju Liu, Department of Gastroenterology, Shanghai Tenth People's Hospital, Clinical Medical College of Nanjing Medical University, 301 Yanchang Middle Road, Zhabei, Shanghai 200072, P.R. China

E-mail: liuzhanju88@126.com

Key words: ulcerative colitis, circular RNA, ATPase class II type 9B, PTEN, apoptosis
circAtp9b suppressed apoptosis. Moreover, overexpression of PTEN reduced the effects of silencing of circAtp9b. In conclusion, overexpression of circAtp9b in UC was induced by LPS and it positively upregulated PTEN to promote the apoptosis of HCnEpCs induced by LPS.

\section{Introduction}

As a type of inflammatory bowel disease that is associated with the formation of ulcers and chronic inflammation in digestive tract, ulcerative colitis (UC) mainly affects the rectum and large intestines (1). In the majority of cases, UC is caused by severe infections, colon wall invasion with lymphocytic white blood cells or collagen, and reduced blood supply to the colon $(2,3)$. Currently, the treatment of UC mainly focuses on the relief of inflammation and improvement of the immune system; however, there is no cure for UC and recurrence is common $(3,4)$. Without timely recovery, long-term existence of UC significantly increases the risk of colorectal cancer, which has a high mortality $(5,6)$. Therefore, more effective treatments for UC are needed.

It has been well established that the development and progression of UC involves the regulation of various molecular pathways, such as the inflammatory pathways and NFKBIZ pathway $(7,8)$. Increased understanding of the molecular mechanisms of UC would facilitate the development of novel therapies, such as targeted therapy that can be used to treat UC by regulating gene expression $(9,10)$. However, to the best of our knowledge, effective targets for UC remain lacking. Circular RNAs (circRNAs/circ) are covalently closed RNA transcripts (single-stranded) that encode no proteins but regulate gene expression to participate in human diseases, suggesting that circRNAs are promising targets for UC targeted therapy $(11,12)$. circ-ATPase class II type 9B (Atp9b) is a newly identified circRNA with enhancing effects on LPS-induced inflammation (13), which contributes to the development of UC (14). Our preliminary deep sequencing analysis results (Li et al, unpublished data) revealed altered expression of circAtp9b and its inverse association with PTEN, which is also a major regulator 
in UC. The present study was therefore carried out to study the potential interaction between circAtp9b and PTEN in UC.

\section{Materials and methods}

Blood sample acquisition. Blood $(3 \mathrm{ml})$ was drawn from 60 patients with UC (34 males and 26 females) and 60 healthy controls ( 34 males and 26 females) under fasting conditions prior to medical or surgical therapy. All patients and healthy controls were admitted at The Affiliated Changzhou No.2 People's Hospital of Nanjing Medical University (Changzhou, China) from March 2019 to May 2020. The age range of the 60 patients with UC was 26-57 years, with a median age of 42 . The age range of the 60 healthy controls was $26-57$ years, with a median age of 42.5. Patients with other severe clinical disorders, including severe infections, diabetes, heart diseases and cancer) or initiated therapy, were excluded from the present study. All of the 60 healthy controls passed systemic physiological exam at the aforementioned hospital. The current study was approved by The Ethics Committee of The Affiliated Changzhou No.2 People's Hospital of Nanjing Medical University (approval no. NMU-2019-13), and was performed in accordance with the ethical standards formulated in the Helsinki Declaration of 1964. All participants signed written informed consent forms.

Plasma preparation and human colonic epithelial cells $(H C n E p C s)$. To isolate plasma from the blood samples, blood samples were transferred to centrifuge tubes containing citrate (1:10 volume ratio). Afterwards, the mixture was centrifuged at $200 \mathrm{x} \mathrm{g}$ for $15 \mathrm{~min}$ at $4^{\circ} \mathrm{C}$ to collect the supernatant. A total of $\sim 1 \mathrm{ml}$ plasma was obtained from each blood sample. HCnEpCs (Cell Applications, Inc.) were used as the cell model of UC. Cells were cultivated in colonic epithelial cell medium (ScienCell Research Laboratories, Inc.) with 5\% $\mathrm{CO}_{2}$ and 95\% humidity in an incubator at $37^{\circ} \mathrm{C}$. For lipopolysaccharide (LPS) treatment, $\mathrm{HCnEpCs}$ were cultivated in medium containing 0 , 10, 20, 50 and $100 \mathrm{ng} / \mathrm{ml}$ LPS (Sigma-Aldrich; Merck KGaA) for $48 \mathrm{~h}$ before use in subsequent experiments.

Transient transfections. Vectors expressing circAtp9b or PTEN were constructed using pcDNA3.1 as the backbone vector (Invitrogen; Thermo Fisher Scientific, Inc.). Scrambled negative control (NC; 5'-GGGAAATTTAAATTACGCCGG-3') and circAtp9b small interfering (si)RNA (5'-GTTCAATGTA GGAACGAGCAT-3') were purchased from Invitrogen (Thermo Fisher Scientific, Inc.). HCnEpCs were transfected with $1 \mu \mathrm{g}$ expression vector or $40 \mathrm{nM}$ siRNA using Lipofectamine ${ }^{\circledR} 2000$ (Invitrogen; Thermo Fisher Scientific, Inc.). To perform NC experiments, empty vector or NC siRNA was transfected into HCnEpCs. HCnEpCs without transfections were cultivated in fresh medium until the end of transfections and used as the control. Cell transfection was performed at $37^{\circ} \mathrm{C}$ for $6 \mathrm{~h}$. Cells were then transferred to fresh medium for further culture. Subsequent experiments were performed at $48 \mathrm{~h}$ after cell transfection.

$R N A$ preparation and reverse transcription-quantitative PCR $(R T-q P C R)$. Total RNA was extracted from plasma samples and the transfected and control HCnEpCs using TRIzol ${ }^{\circledR}$ (Invitrogen; Thermo Fisher Scientific, Inc.). RNA samples were digested with DNase I (Invitrogen; Thermo Fisher Scientific, Inc.) at $37^{\circ} \mathrm{C}$ for 80 min to remove genomic DNA. Electrophoresis was carried out using 5\% urea-PAGE gel to analyze RNA integrity. OD260/280 ratios were checked to determine RNA purity. RNA samples were reverse transcribed into cDNA samples using a SuperScript ${ }^{\mathrm{TM}}$ III RT system (Invitrogen; Thermo Fisher Scientific, Inc.). The thermocycling conditions were as follows: $37^{\circ} \mathrm{C}$ for $60 \mathrm{~min} ; 85^{\circ} \mathrm{C}$ for $5 \mathrm{~min}$; $4^{\circ} \mathrm{C}$ for $5 \mathrm{~min}$. With cDNA samples as template, qPCR reactions were prepared using a QuantiTect SYBR ${ }^{\circledR}$-Green PCR kit (Qiagen, Inc.). The thermocycling conditions were as follows: Initial denaturation at $95^{\circ} \mathrm{C}$ for $2 \mathrm{~min}$, followed by 40 cycles of denaturation at $95^{\circ} \mathrm{C}$ for $15 \mathrm{sec}$ and annealing at $60^{\circ} \mathrm{C}$ for $45 \mathrm{sec}$, with a final extension at $72^{\circ} \mathrm{C}$ for $10 \mathrm{~min}$. The expression levels of circAtp9b and PTEN were determined with GAPDH as an internal control. Three technical replicates were performed and the $2^{-\Delta \Delta C q}$ method was used for the normalization of $\mathrm{Cq}$ values (15). The primer sequences were listed in Table I.

Western blotting. Total proteins were isolated from the transfected and control HCnEpCs using RIPA lysis buffer(Invitrogen; Thermo Fisher Scientific, Inc.). Protein quantification was performed using a BCA assay (Invitrogen; Thermo Fisher Scientific, Inc.). Protein samples (10 $\mu \mathrm{g} / \mathrm{lane})$ were separated via SDS-PAGE on $8 \%$ gels and then transferred onto PVDF membranes. The membranes were blocked in PBS containing $5 \%$ non-fat milk at room temperature for $2 \mathrm{~h}$. After that, the membranes were incubated with rabbit PTEN (1:500; cat. no. ab31392; Abcam) and mouse GAPDH (1:2,000; cat. no. ab8245; Abcam) primary antibodies at $4^{\circ} \mathrm{C}$ for $18 \mathrm{~h}$, followed by incubation with a goat anti-rabbit IgG-HRP secondary antibody (1:1,000; cat. no. MBS435036; MyBioSource, Inc.) or a goat anti-mouse IgG-HRP secondary antibody $(1: 10,000$; cat. no. ab6789; Abcam) at room temperature for $3 \mathrm{~h}$. Signals were produced using the Western Lightning Plus-ECL kit (PerkinElmer, Inc.). Data quantification was performed using ImageJ v1.44 software (National Institutes of Health).

Apoptosis assay. Transfected HCnEpCs were transferred to six-well cell culture plates with $2 \mathrm{ml}$ fresh medium per well. LPS $(100 \mathrm{ng} / \mathrm{ml})$ was added to each well, followed by cell culture at $37^{\circ} \mathrm{C}$ for $48 \mathrm{~h}$. After that, cells were collected and resuspended in binding buffer followed by staining with FITC-Annexin V and propidium iodide (Abcam) for $15 \mathrm{~min}$ in the dark at room temperature. Apoptotic cells were detected using a FACSCalibur flow cytometer (BD Biosciences) and its supplied software (BD CellQuest software). The apoptotic rate was considered to be equal to the percentage of early and late apoptotic cells.

Statistical analysis. Statistical analysis was performed using SPSS v22.0 software (IBM Corp.). Gene expression levels in the plasma samples from patients with $\mathrm{UC}(\mathrm{n}=60)$ and controls $(n=60)$ were expressed as the mean values of 3 technical replicates, and an unpaired t-test was used for data comparison. Mean \pm standard deviation values were used to express the data of 3 biological replicates of cell transfection experiments, and ANOVA with Tukey's post hoc test was used for data comparisons among the groups. Correlations were analyzed by Pearson's correlation coefficient. $\mathrm{P}<0.05$ was considered to indicate a statistically significant difference. 
Table I. Primers for reverse transcription-quantitative PCR.

\begin{tabular}{lll}
\hline Gene & \multicolumn{1}{c}{ Forward primers, 5'-3' } & Reverse primers, 5'-3' \\
\hline PTEN & AAGACCATAACCCACCACAGC & CACCAGTTCGTCCCTTTCCA \\
circAtp9b & AGGCCTTTCTGTCTTGTGGT & AATGGGCAGACTCATCCTCC \\
GAPDH & CTTCGGCATTGTGGAGGG & GGAGGCAGGGATGATGTTCT
\end{tabular}

circ, circular RNA; Atp9b, ATPase class II type 9B.
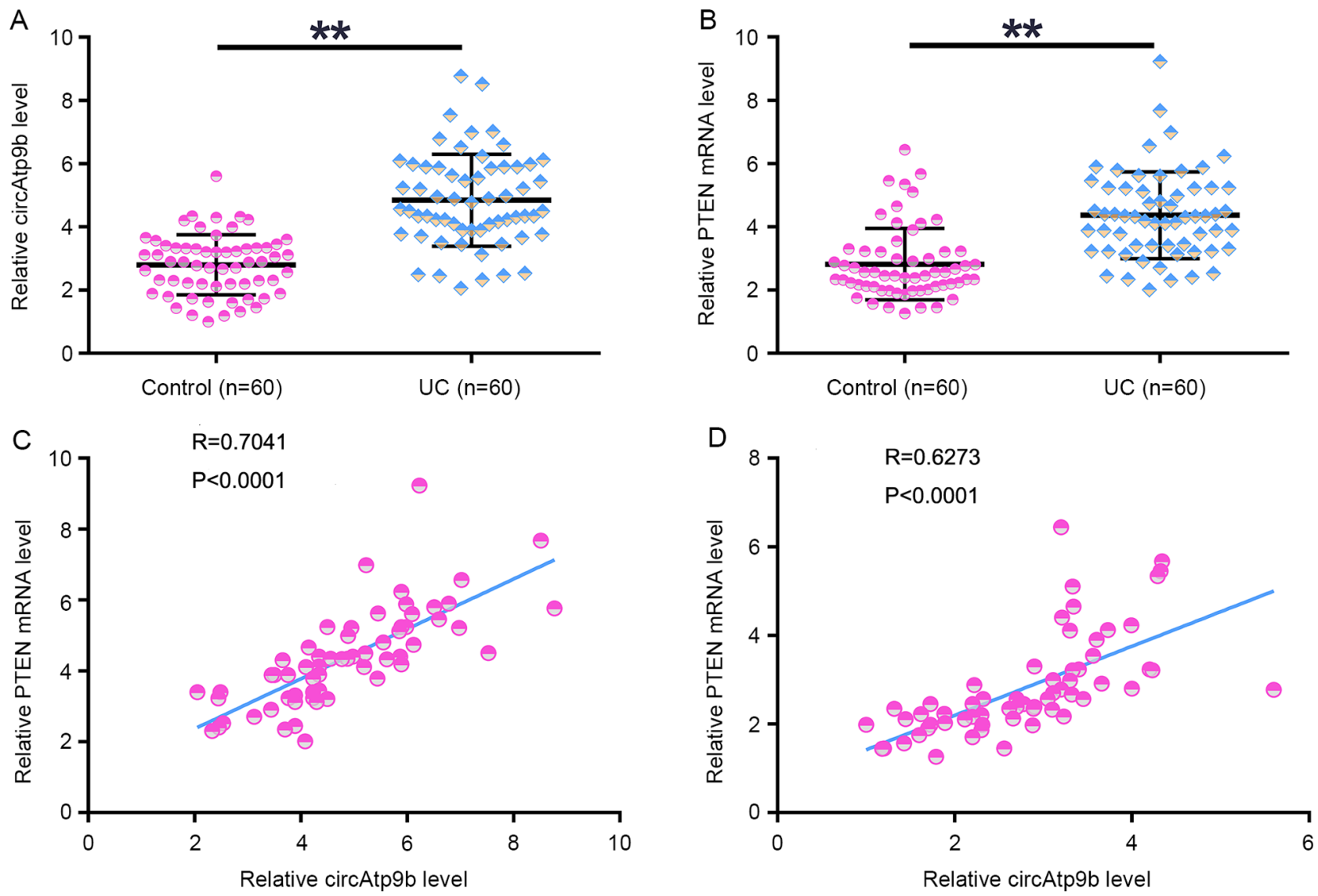

Figure 1. Expression of circAtp9b and PTEN is positively correlated in patients with UC. Expression levels of (A) circAtp9b and (B) PTEN mRNA in plasma samples collected from patients with UC $(n=60)$ and healthy controls $(n=60)$ were analyzed by performing reverse transcription-quantitative PCR. Pearson's correlation coefficient analysis was performed to analyze the correlation between circAtp9b and PTEN mRNA in both (C) UC and (D) control plasma samples. ${ }^{* *} \mathrm{P}<0.01$. UC, ulcerative colitis; circ, circular RNA; Atp9b, ATPase class II type 9B.

\section{Results}

Expression of circAtp9b and PTEN is positively correlated in patients with UC. The expression levels of circAtp9b and PTEN in the plasma samples collected from patients with UC $(n=60)$ and healthy controls $(n=60)$ were determined using RT-qPCR. It was observed that the expression levels of circAtp9b (Fig. 1A) and PTEN (Fig. 1B) were significantly upregulated in patients with UC compared with those in the controls (both $\mathrm{P}<0.01$ ). Correlation analysis revealed that the expression levels of circAtp9b and PTEN were significantly positively correlated with each other across both UC (Fig. 1C) and control (Fig. 1D) plasma samples. Therefore, circAtp9b and PTEN may interact with each other under both physiological and pathological conditions.
LPS treatment increases the expression levels of circAtp9b and PTEN in HCnEpCs. HCnEpCs were cultivated in medium containing $0,10,20,50$ and $100 \mathrm{ng} / \mathrm{ml}$ LPS for $48 \mathrm{~h}$, followed by measuring the expression levels of circAtp9b and PTEN via RT-qPCR. It was observed that LPS treatment significantly increased the expression levels of circAtp9b $(\mathrm{P}<0.05$; Fig. $2 \mathrm{~A})$ and PTEN $(\mathrm{P}<0.05$; Fig. $2 \mathrm{~B})$ in a dose-dependent manner. These results indicated that circAtp9b and PTEN were LPS-inducible.

circAtp9b positively regulates the $m R N A$ and protein expression levels of PTEN in HCnEpCs. Overexpression vectors for circAtp9b or PTEN, or circAtp9b siRNA were transfected into $\mathrm{HCnEpCs}$ to explore the relationship between PTEN and circAtp9b. Transfections were confirmed via RT-qPCR at $48 \mathrm{~h}$ post-transfection $(\mathrm{P}<0.05$; Fig. $3 \mathrm{~A})$. Overexpression 


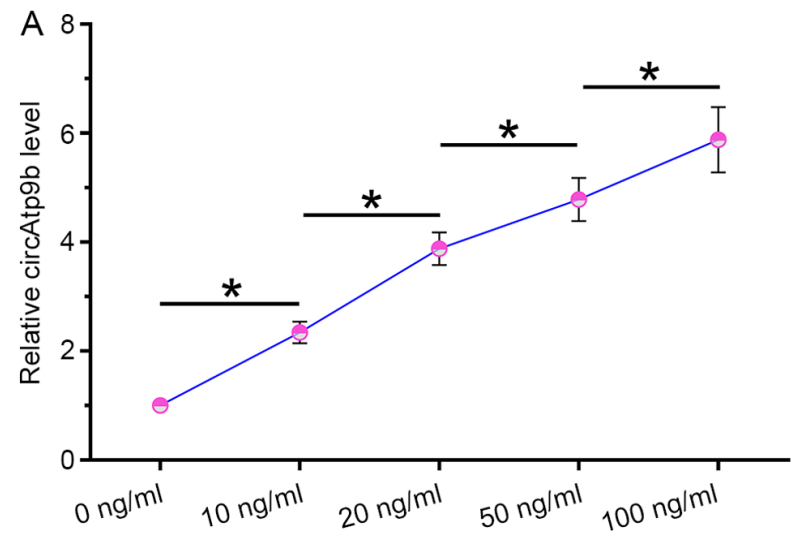

LPS

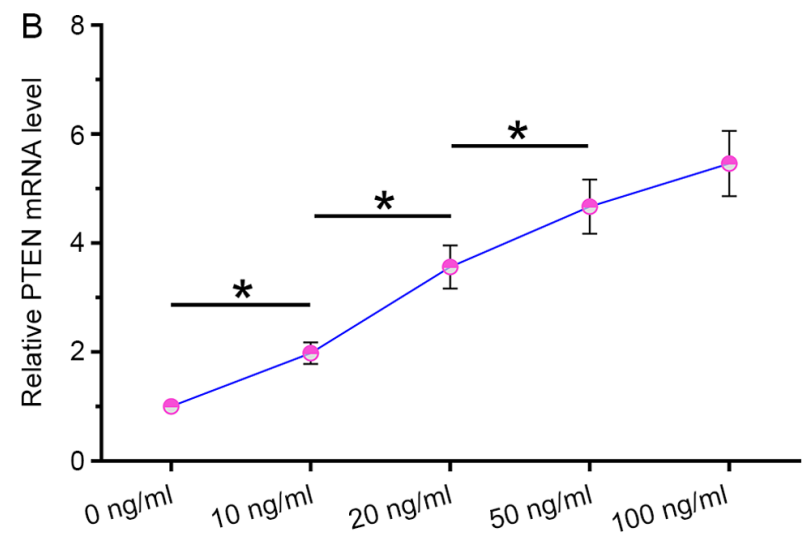

LPS

Figure 2. LPS treatment increased the expression of both circAtp9b and PTEN mRNA in HCnEpCs. HCnEpCs were cultivated in medium containing $0,10,20$, 50 or $100 \mathrm{ng} / \mathrm{ml}$ LPS for $48 \mathrm{~h}$, followed by the measurement of the expression levels of (A) circAtp $9 \mathrm{~b}$ and (B) PTEN mRNA by reverse transcription-quantitative PCR. "P<0.05. LPS, lipopolysaccharides; circ, circular RNA; Atp9b, ATPase class II type 9B; HCnEpCs, human colonic epithelial cells.
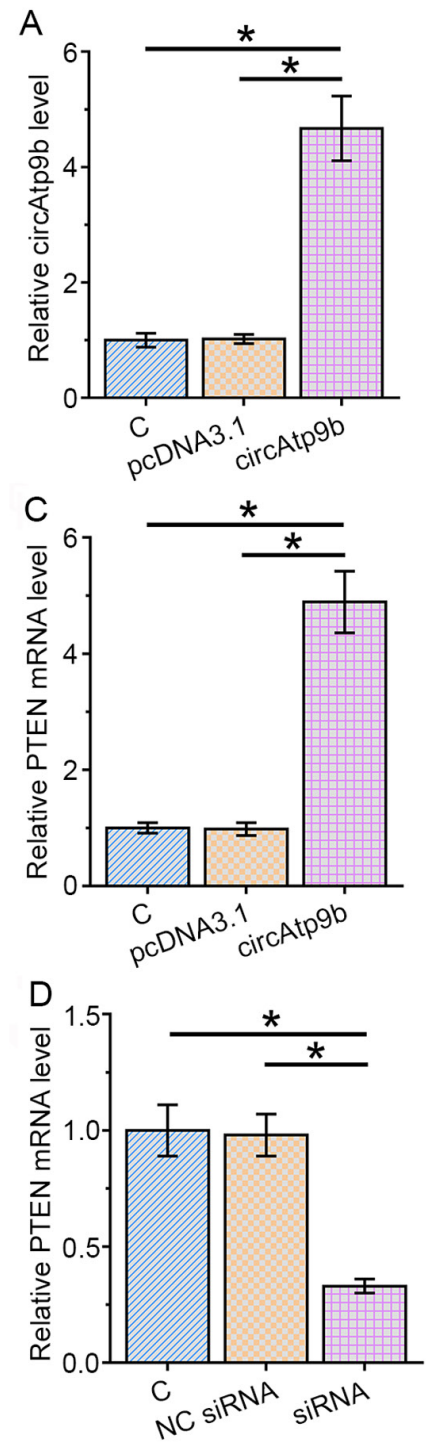
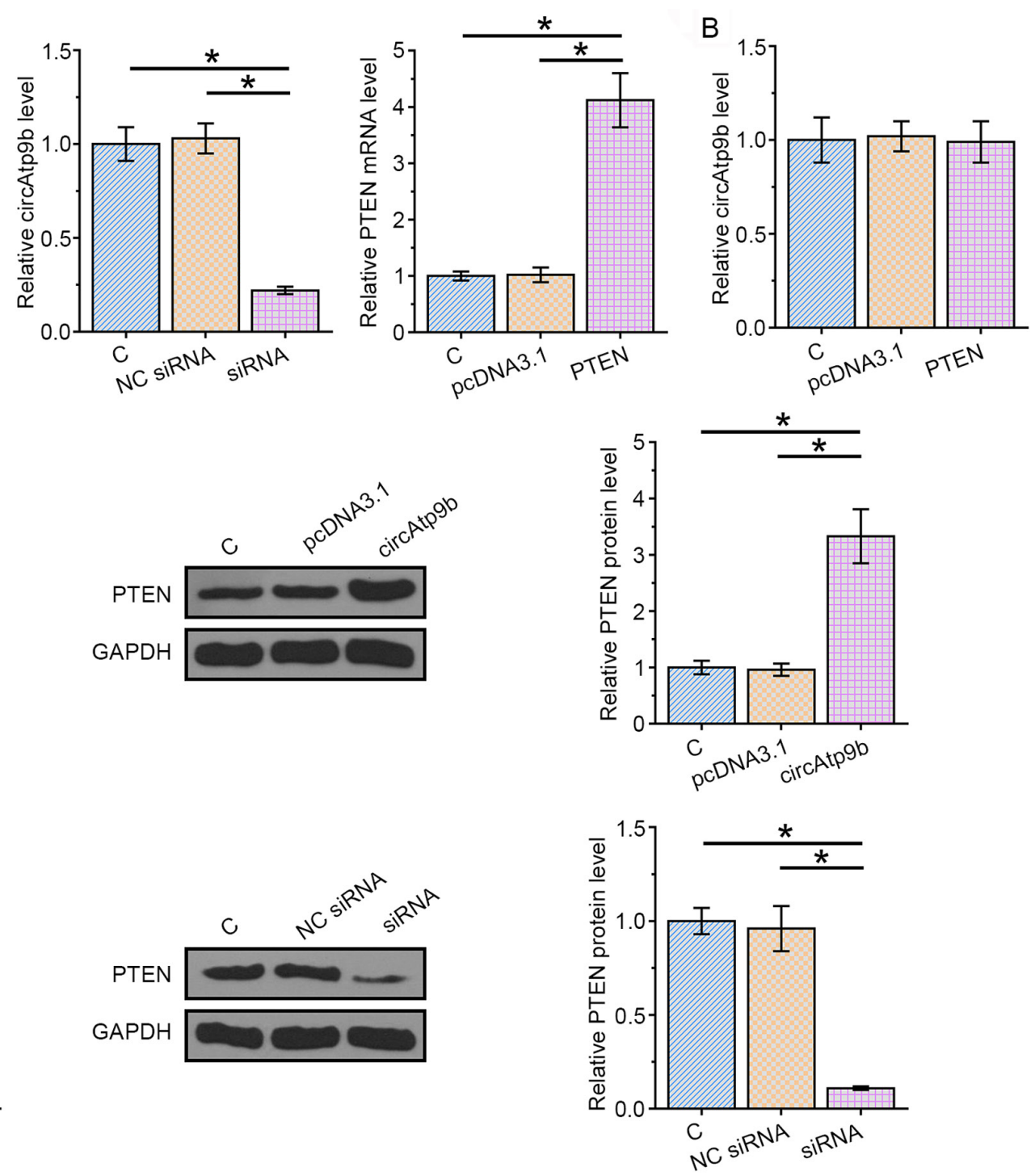

Figure 3. circAtp9b positively regulates the expression levels of PTEN in HCnEpCs at both mRNA and protein levels. Expression vectors of circAtp9b or PTEN, or circAtp9b siRNA were transfected into HCnEpCs to analyze the relationship between PTEN and circAtp9b. (A) Transfections were confirmed by RT-qPCR at $48 \mathrm{~h}$ post-transfection. (B) RT-qPCR was performed to analyze the effects of PTEN overexpression on the expression of circAtp9b. Effects of (C) circAtp9b overexpression and (D) siRNA-mediated silencing on the expression of PTEN were analyzed by RT-qPCR and western blotting. "P<0.05. RT-qPCR, reverse transcription-quantitative PCR; circ, circular RNA; Atp9b, ATPase class II type 9B; siRNA, small interfering RNA; HCnEpCs, human colonic epithelial cells; $\mathrm{NC}$, negative control; C, control. 

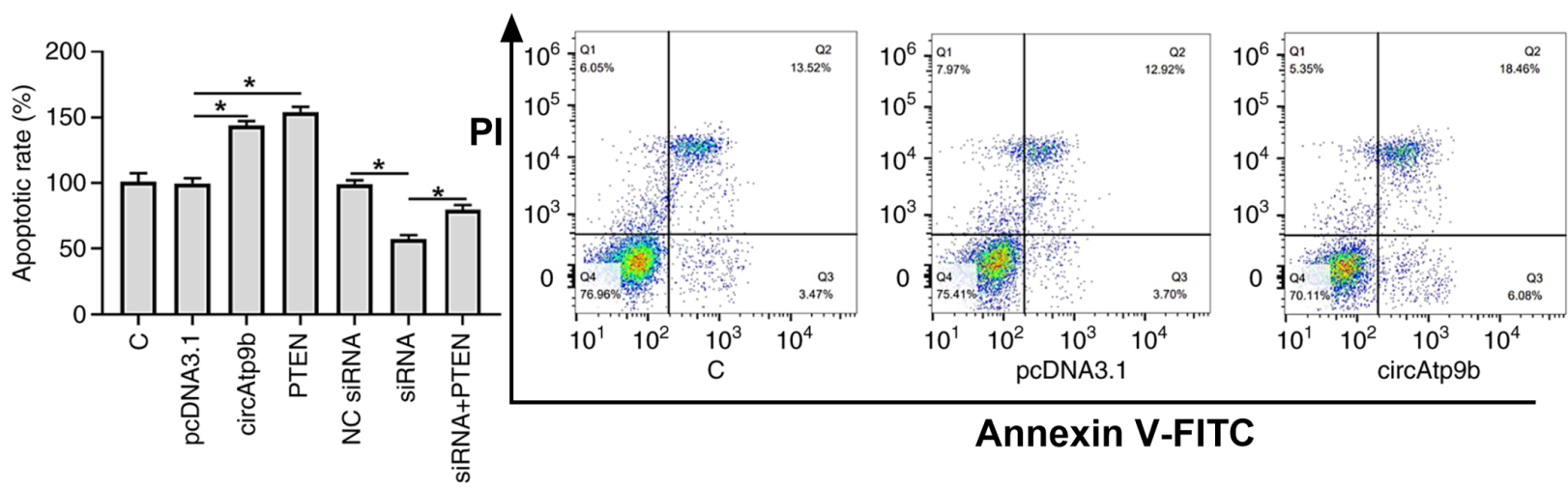

\section{Annexin V-FITC}
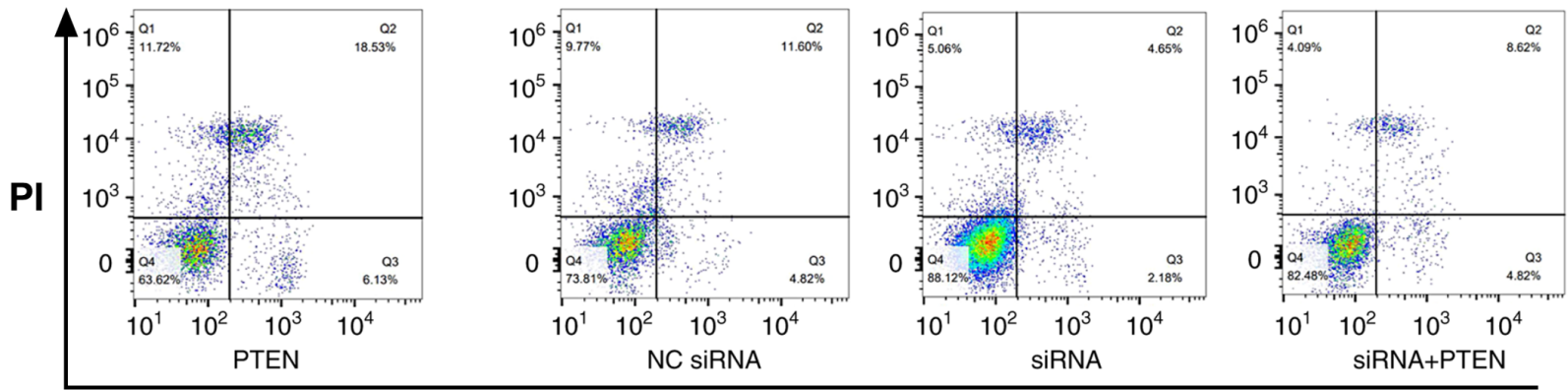

Annexin V-FITC

Figure 4. circAtp9b promotes the LPS-induced apoptosis of HCnEpCs via PTEN. LPS-induced HCnEpCs were treated with a circAtp9b overexpression vector, PTEN overexpression vector or circAtp9b siRNA. Apoptosis was detected using FITC-Annexin V. "P<0.05. circ, circular RNA; Atp9b, ATPase class II type 9B; HCnEpCs; LPS, lipopolysaccharides; siRNA, small interfering RNA; C, control; NC, negative control.

of PTEN did not significantly affect the expression of circAtp9b (Fig. 3B). The expression levels of PTEN in cells transfected with circAtp9b expression vector or circAtp9b siRNA were determined by RT-qPCR and western blotting. It was observed that overexpression of circAtp9b significantly mediated the upregulation of PTEN in HCnEpCs compared with the control ( $\mathrm{P}<0.05$; Fig. 3C), whereas silencing circAtp9b decreased the expression levels of PTEN ( $<<0.05$; Fig. 3D). Therefore, circAtp9b was likely an upstream activator of PTEN in HCnEpCs.

circAtp9b promotes the LPS-induced apoptosis of HCnEpCs via PTEN. The role of circAtp9b and PTEN in regulating HCnEpC apoptosis induced by LPS treatment $(100 \mathrm{ng} / \mathrm{ml}$ for $48 \mathrm{~h}$ ) was assessed via flow cytometry. As presented in Fig. 4, overexpression of circAtp9b and PTEN significantly promoted the apoptosis of HCnEpCs compared with the empty vector control $(\mathrm{P}<0.05)$, while knockdown of circAtp9b significantly inhibited the apoptosis of HCnEpCs compared with the NC siRNA $(\mathrm{P}<0.05)$. However, the effects of knocking down circAtp9b on apoptosis were significantly attenuated by overexpression of PTEN $(\mathrm{P}<0.05)$. These results suggested that circAtp9b promoted LPS-induced apoptosis of HCnEpCs by regulating PTEN.

\section{Discussion}

The present study mainly explored the involvement of circAtp9b in UC and its potential interactions with PTEN. It was revealed that circAtp9b was significantly upregulated in $\mathrm{UC}$ and it may positively regulate the expression of PTEN to promote the apoptosis of HCnEpCs induced by LPS.
circRNAs have been demonstrated to have notable roles in numerous types of disease, but their roles in UC remain largely unknown (11). In a recent study, it was reported that the expression of circ-superoxide dismutase 2 (SOD2) was altered in $\mathrm{UC}$ and regulated the intestinal epithelial barrier (12). In another study, circRNA hsa_circ_0007919 was indicated to be significantly upregulated in UC and is likely associated with the pathogenesis of UC (16). However, the role of circRNAs circ-SOD2 and hsa_circ_0007919 in UC remains unclear $(11,16)$. It was reported that circAtp9b has an enhancing effect on LPS-induced inflammation (13). It has been well established that LPS-induced inflammation contributes to the development of UC (14), suggesting the involvement of circAtp9b in UC. The present study demonstrated that circAtp9b is upregulated in UC. In addition, LPS treatment increased the expression levels of circAtp9b in $\mathrm{HCnEpCs}$ in a dose-dependent manner, and circAtp9b positively regulated the apoptosis of $\mathrm{HCnEpCs}$ induced by LPS. Therefore, circAtp9b may promote UC through LPS-dependent pathways.

PTEN promotes apoptosis (17), which contributes to the aggregation of UC (18). The present study observed the upregulation of PTEN in UC, and that it had an enhancing effect on the apoptosis of HCnEpCs induced by LPS. LPS may indirectly regulate the expression of PTEN through microRNAs (19). Consistently, the present study demonstrated increased expression levels of PTEN in LPS-treated HCnEpCs. circRNAs mainly participate in biological processes by regulating gene expression at both transcriptional and translational levels (11). The present study revealed that circAtp9b positively regulated the expression of PTEN in HCnEpCs, while overexpression of PTEN did not affect the expression of circAtp9b. Therefore, circAtp9b is likely 
an upstream activator of PTEN. Crohn's disease and UC are two forms of inflammatory bowel diseases. The PI3K/AKT/PTEN pathway acts as a pivotal determinant of cell fate regarding senescence and apoptosis, which is mediated by intracellular reactive oxygen species (ROS) generation in Crohn's disease (20). Therefore, the mechanism of HCnEpCs apoptosis may be associated with the upregulation of the PI3K/AKT/PTEN pathway and the generation of intracellular ROS.

However, the present study has certain limitations. Firstly, the sample size of patients with UC was limited. Future studies should include more samples to strengthen the findings. In addition, the current study did not uncover the molecular mechanisms via which circAtp9b regulates the expression of PTEN, and further studies are required to investigate the implicated mechanisms.

In conclusion, circAtp9b and PTEN were both upregulated in UC. In addition, circAtp9b could positively regulate the expression of PTEN to promote the apoptosis of HCnEpCs induced by LPS.

\section{Acknowledgements}

Not applicable.

\section{Funding}

No funding was received.

\section{Availability of data and materials}

The datasets used and/or analyzed during the current study are available from the corresponding author on reasonable request.

\section{Authors' contributions}

FL and XW confirm the authenticity of all the raw data. FL and JF performed the clinical studies, experimental work, data analysis and manuscript writing. LF, SL and HZ prepared the manuscript, and performed the experimental work and literature research. XW and ZL conceived the study and designed the research, and edited the manuscript. All authors have read and approved the final manuscript.

\section{Ethics approval and consent to participate}

Ethical approval was obtained from The Ethics Committee of the Affiliated Changzhou No.2 People's Hospital of Nanjing Medical University (approval no. NMU-2019-13). Informed consent was signed by all participants.

\section{Patient consent for publication}

Not applicable.

\section{Competing interests}

The authors declare that they have no competing interests.

\section{References}

1. Rubin DT, Ananthakrishnan AN, Siegel CA, Sauer BG and Long MD: ACG clinical guideline: Ulcerative colitis in adults. Am J Gastroenterol 114: 384-413, 2019.

2. Testa A,Castiglione F, Nardone OM and Colombo GL: Adherence in ulcerative colitis: An overview. Patient Prefer Adherence 11: 297-303, 2017.

3. Hindryckx P, Jairath V and D'Haens G: Acute severe ulcerative colitis: From pathophysiology to clinical management. Nat Rev Gastroenterol Hepatol 13: 654-664, 2016.

4. Null K, Xu Y, Pasquale M, Su C, Marren A, Harnett J, Mardekian J, Manuchehri A and Healey P: Ulcerative colitis treatment patterns and cost of care. Value Health 20: 752-761, 2016.

5. Olén O, Erichsen R, Sachs MC, Pedersen L, Halfvarson J, Askling J, Ekbom A, Sørensen HT and Ludvigsson JF: Colorectal cancer in ulcerative colitis: A Scandinavian population-based cohort study. Lancet 395: 123-131, 2020.

6. Damas OM and Abreu MT: Are patients with ulcerative colitis still at increased risk of colon cancer? Lancet 395: 92-94, 2020.

7. Tatiya-Aphiradee N, Chatuphonprasert W and Jarukamjorn K: Immune response and inflammatory pathway of ulcerative colitis. J Basic Clin Physiol Pharmacol 30: 1-10, 2018.

8. Kakiuchi N, Yoshida K, Uchino M, Kihara T, Akaki K, Inoue Y, Kawada K, Nagayama S, Yokoyama A, Yamamoto S, et al: Frequent mutations that converge on the NFKBIZ pathway in ulcerative colitis. Nature 577: 260-265, 2020.

9. Zhang Q, Tao H, Lin Y, Hu Y, An H, Zhang D, Feng S, Hu H, Wang R, Li X, et al: A superoxide dismutase/catalase mimetic nanomedicine for targeted therapy of inflammatory bowel disease. Biomaterials 105: 206-221, 2016.

10. Allocca M, Furfaro F, Fiorino G, Gilardi D, D'Alessio S and Danese S: Can IL-23 be a good target for ulcerative colitis? Best Pract Res Clin Gastroenterol 32-33: 95-102, 2018.

11. Ebbesen KK, Hansen TB and Kjems J: Insights into circular RNA biology. RNA Biol 14: 1035-1045, 2017.

12. Wang TT, Han Y, Gao FF, Ye L and Zhang YJ: Effects of circular RNA circ-SOD2 on intestinal epithelial barrier and ulcerative colitis. Beijing Da Xue Xue Bao Yi Xue Ban 51: 805-812, 2019 (In Chinese).

13. Sun J, Wang X, Wang D, Zhao Z, Zhang L and Zhang J: circAtp9b knockdown alleviates LPS-caused inflammation provided that microRNA-27a is upregulated. Int Immunopharmacol 78: $105925,2020$.

14. Obana N, Takahashi S, Kinouchi Y, Negoro K, Takagi S, Hiwatashi $\mathrm{N}$ and Shimosegawa T: Ulcerative colitis is associated with a promoter polymorphism of lipopolysaccharide receptor gene, CD14. Scand J Gastroenterol 37: 699-704, 2002.

15. Livak KJ and Schmittgen TD: Analysis of relative gene expression data using real-time quantitative PCR and the $2(-\Delta \Delta \mathrm{C}(\mathrm{T}))$ method. Methods 25: 402-408, 2001.

16. Wang T, Chen N, Ren W, Liu F, Gao F, Ye L, Han Y, Zhang Y and Liu Y: Integrated analysis of circRNAs and mRNAs expression profile revealed the involvement of hsa_circ_0007919 in the pathogenesis of ulcerative colitis. J Gastroenterol 54: 804-818, 2019.

17. Yamada KM and Araki M: Tumor suppressor PTEN: Modulator of cell signaling, growth, migration and apoptosis. J Cell Sci 114: 2375-2382, 2001

18. Li Z, Liu GX, Liu YL, Chen X, Huang XL and Gan HT: Effect of adenovirus-mediated PTEN gene on ulcerative colitis-associated colorectal cancer. Int J Colorectal Dis 28: 1107-1115, 2013.

19. Liu J, Xu D, Wang Q, Zheng D, Jiang X and Xu L: LPS induced miR-181a promotes pancreatic cancer cell migration via targeting PTEN and MAP2K4. Dig Dis Sci 59: 1452-1460, 2014.

20. Tokuhira, Kitagishi Y, Suzuki M, Minami A, Nakanishi A, Ono Y, Kobayashi K, Matsuda S and Ogura Y: PI3K/AKT/PTEN pathway as a target for Crohn's disease therapy (Review). Int J Mol Med 35: 10-16, 2015. (i) $\Theta$ This work is licensed under a Creative Commons Attribution-NonCommercial-NoDerivatives 4.0 International (CC BY-NC-ND 4.0) License. 